CUBO A Mathematical Journal

Vol.22, $N^{-} 01$, (137-154). April 2020

\title{
Level sets regularization with application to optimization problems
}

\author{
Moussa Barro and Sado Traore \\ Université Nazi BONI, \\ Burkina Faso. \\ mousbarro@yahoo.fr, traore.sado@yahoo.fr
}

\begin{abstract}
Given a coupling function $c$ and a non empty subset of $\mathbb{R}$, we define a closure operator. We are interested in extended real-valued functions whose sub-level sets are closed for this operator. Since this class of functions is closed under pointwise suprema, we introduce a regularization for extended real-valued functions. By decomposition of the closure operator using polarity scheme, we recover the regularization by bi-conjugation. We apply our results to derive a strong duality for a minimization problem.
\end{abstract}

\section{RESUMEN}

Dada una función de acoplamiento $c$ y un subconjunto no vacío de $\mathbb{R}$, definimos un operador clausura. Estamos interesados en funciones extendidas a valores reales cuyos conjuntos de sub-nivel son cerrados para este operador. Dado que esta clase de funciones es cerrada bajo supremos puntuales, introducimos una regularización para funciones extendidas a valores reales. Gracias a la descomposición del operador clausura usando el esquema de polaridad, recuperamos la regularización por bi-conjugación. Aplicamos nuestros resultados para derivar una dualidad fuerte para un problema de minimización.

Keywords and Phrases: Duality, regularization, level sets, c-elementary functions, polarity, conjugacy.

2010 AMS Mathematics Subject Classification: 49N15. 


\section{Introduction}

Regularization and conjugation of extended real-valued functions play an important role in optimization theory since it is a base of duality theory. Until the Fenchel's work(4), many authors have introduced and studied different kinds of regularization and conjugation among which we can cite Moreau ([8]), Crouzeix ([1]), Rockafellar ([13]), Martínez-Legaz ([7]), Singer ([15]), Penot-Volle ([1]), Volle ([16, 17]). In [16, M. Volle used a dual pair of polarities to introduce and study level set regularization and conjugacy.

In this work, we introduce and study level set regularization and conjugacy by means of a coupling function and a nonvoid subset of the real numbers. The outline of the paper is as follows. In Section 2 we recall Moreau conjugation scheme. Section 3 is devoted to the study of the $\Gamma$-regularization of extended real-valued functions and hull of sets. We introduce these notions and give some properties (Proposition 2, 4 and Theorem 3.8). In Section 4, we introduce the level set regularization of extended real-valued functions. By decomposition of a closure operator via a couple of dual polarities, we show that this regularization coincides with the bi-conjugation relative to the polarity couple (Proposition 8 and Theorem 4.6). We derive an analytic expression of level set regularization of extended real-valued functions (Proposition 10). Section 5 is devoted to an application of our theory to a minimization problem. A perturbational dual of this problem is defined and a necessary and sufficient condition is given to ensure a strong duality property for this problem (Theorem 5.1. Corollary 5.2 and Corollary 5.3).

\section{Preliminaries}

Let us start this section by recalling the Moreau conjugation (8]). Let $U, V$ two nonvoid sets and $c: U \times V \rightarrow \mathbb{R}$, a coupling function. Given an extended real-valued function $h: U \rightarrow \overline{\mathbb{R}}:=$ $\mathbb{R} \cup\{-\infty,+\infty\}$, we define the $c-$ conjugate of $h$ by $h^{c}(v):=\sup _{u \in U}\{c(u, v)-h(u)\}$, for any $v \in V$. By exchanging the role of $U$ and $V$, we define the $c$-conjugate of a given function $k: V \rightarrow \overline{\mathbb{R}}$ by $k^{c}(u):=\sup _{v \in V}\{c(u, v)-k(v)\}$ for any $u \in U$. The $c-$ bi-conjugate of a given $h: U \rightarrow \overline{\mathbb{R}}$ is then defined by $h^{c c}(u):=\sup _{v \in V}\left\{c(u, v)-h^{c}(v)\right\}$ for any $u \in U$.

Example 2.1. The usual Legendre-Fenchel conjugacy is obtained by taking $U:=X$, a topological vector space with topological dual $V:=X^{*}$ and $c$ the standard bilinear coupling function.

Example 2.2. Other examples of coupling functions have been considered in the literature among which, we can cite:

(1) $U=V=\left\{x \in \mathbb{R}^{n} \mid x_{1}>0, \ldots, x_{n}>0\right\}$ and $c(u, v)=\min _{1 \leq i \leq n} v_{i} u_{i}$, (14]), 
(2) $(U, d)$ a metric space, $\alpha>0, V=U$ and $c(u, v)=-\alpha d(u, v)$, ([G] $)$,

(3) $(U, d)$ a metric space, $V=U \times] 0,+\infty[$ and $c(u,(v, \alpha))=-\alpha d(u, v)$, ([] $]$ ),

(4) $U=V=\mathbb{R}^{n}, 0<\alpha \leq 1, \beta>0$ and $c(u, v)=-\beta\|u-v\|^{\alpha}$, ([7]),

(5) $U$ a topological space, $V=\mathscr{C}(U, \mathbb{R})$, space of countinuous real functions on $U$ and $c(u, v)=$ $v(u),([5],[10])$.

Given a function $h: U \rightarrow \overline{\mathbb{R}}$, the following notation and definitions will be needed: dom $h=$ $\{u \in U \mid h(u)<+\infty\}$, the effective domain of $h,[h \leq t]:=\{u \in U \mid h(u) \leq t\}$, the $t$-sub-level set of $h$ (or level set of $h$ in short).

Given a subset $A$ of $U$, we define its indicator function $i_{A}$ by $i_{A}(u)=0$ if $u \in A$ and $i_{A}(u)=$ $+\infty$ if $u \in U \backslash A$. Following the terminology introduced in [9] we will also use the valley function $v_{A}$ of $A$ defined by $v_{A}(u)=-\infty$ if $u \in A$ and $v_{A}(u)=+\infty$ if $u \in U \backslash A$.

\section{$3 \quad \Gamma$-regularization of functions and hull of sets}

\section{1 $\Gamma$-regularization of functions}

The notion of continuous affine functions can be generalized by those of $c$-elementary functions. In this work, we call $c$-elementary function on $U$ (resp. on $V$ ), the function of the form $c(., v)-r$ (resp. $c(u,)-r$.$) with v \in V$ (resp. $u \in U$ ) and $r \in \mathbb{R}$. The upper hull (i.e., the supremum) of a family of $c$-elementary functions is called $c$-regular. We denote by $\Gamma_{c}(U)$, the set of $c$-regular functions defined on $U$. We call $c$-hull or $\Gamma_{c}$-regularization of $h: U \rightarrow \overline{\mathbb{R}}$, the greatest $c$-regular minorant of $h$. This function is denoted by $h^{\Gamma_{c}}$. It is well known ([8]) that

$$
h^{c c}=h^{\Gamma_{c}} \text {, for each } h: U \rightarrow \overline{\mathbb{R}} \text {. }
$$

Remark 3.1. The equality (3.1) is still valid if the coupling function is an extended real-valued function. In this case, one must interpret the conjugate $h^{c}$ as follows

$$
h^{c}(v)=-\inf _{u \in U}\{h(u)-c(u, v)\},
$$

with the usual conventions $(+\infty)-(+\infty)=(-\infty)-(-\infty)=+\infty$.

There exists an equivalent approach to generalized convex duality in terms of $\Phi$-convexity [2], which consists of working with a set $U$ and a class of functions $\Phi \subset \overline{\mathbb{R}}^{U}$. 


\section{$3.2 \quad$ Hull of sets}

Let $\mathbb{P}$ be a nonvoid subset of $\mathbb{R}$. The following definition generalizes the notion of half space.

Definition 1. We call $(c, \mathbb{P})$ - elementary subset of $U$ any subset of $U$ of the form $\{u \in U \mid r-$ $c(u, v) \in \mathbb{P}\}$, where $(v, r) \in V \times \mathbb{R}$. We note it by $E_{v, r}^{\mathbb{P}}$.

Note that, if $\mathbb{P}=\mathbb{R}$, then $E_{v, r}^{\mathbb{P}}=U$ for any $(v, r) \in V \times \mathbb{R}$. In this case, the only $(c, \mathbb{P})$-elementary subset of $U$ is $U$ itself. The $(c, \mathbb{P})$-elementary subsets of $U$ allow us to define a notion of hull of a subset $A$ of $U$.

Definition 2. The $(c, \mathbb{P})$-hull of $A \subset U$ is the intersection of all $(c, \mathbb{P})$-elementary subsets of $U$ containing $A$. The $(c, \mathbb{P})-$ hull of $A$ is denoted by $\langle A\rangle_{c, \mathbb{P}}$.

Remark 3.2. If there is not $(c, \mathbb{P})$-elementary subset of $U$ containing $A$, then $\langle A\rangle_{c, \mathbb{P}}=U$ by convention.

Proposition 1. If $\mathbb{P} \neq \mathbb{R}$, then $\langle\emptyset\rangle_{c, \mathbb{P}}=\emptyset$.

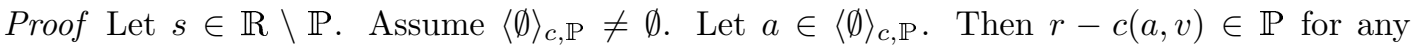
$(v, r) \in V \times \mathbb{R}$. In particular $s=(s+c(a, v))-c(a, v) \in \mathbb{P}$, absurd.

It follows from the definition of $\langle.\rangle_{c, \mathbb{P}}$ that, for each $A \subset U$, and for each $u \in U$, one has

$$
u \notin\langle A\rangle_{c, \mathbb{P}} \Longleftrightarrow \exists(v, r) \in V \times \mathbb{R}: A \subset E_{v, r}^{\mathbb{P}} \text { and } r-c(u, v) \notin \mathbb{P} .
$$

By definition 1, one has $A \subset\langle A\rangle_{c, \mathbb{P}}$, for any $A \subset U$. Moreover, if $A \subset B$ then $\langle A\rangle_{c, \mathbb{P}} \subset\langle B\rangle_{c, \mathbb{P}}$. Therefore, $\left\langle\langle A\rangle_{c, \mathbb{P}}\right\rangle_{c, \mathbb{P}}=\langle A\rangle_{c, \mathbb{P}}, \forall A \subset U$. We deduce that $\langle.\rangle_{c, \mathbb{P}}$ is an algebraic closure operator.

Definition 3. A subset $A$ of $U$ is said to be $(c, \mathbb{P})$-regular if $A=\langle A\rangle_{c, \mathbb{P}}$. We denote $\mathcal{R}_{c, \mathbb{P}}(U)$, the set of all $(c, \mathbb{P})$-regular subsets of $U$.

Observe that $(c, \mathbb{P})$-elementary sets are $(c, \mathbb{P})$-regular. More generally, any intersection of $(c, \mathbb{P})$-regular subsets is $(c, \mathbb{P})$-regular and the $(c, \mathbb{P})$-regular hull of $A \subset U$ coincides with the intersection of all $(c, \mathbb{P})$-regular subsets of $U$ containing $A$.

In what follows, we will use the following values for $\mathbb{P}: \mathbb{P}_{1}=\mathbb{R}_{+}:=\left[0,+\infty\left[, \mathbb{P}_{2}=\mathbb{R}_{+}^{*}:=\right.\right.$ ] $0,+\infty\left[, \mathbb{P}_{3}=\mathbb{R}^{*}:=\mathbb{R} \backslash\{0\}\right.$ and $\mathbb{P}_{4}=\{0\}$. For $i=1,2,3,4,\langle\cdot\rangle_{c, i}:=\langle.\rangle_{c, \mathbb{P}_{i}}$ for short. For $i=1,2,3$, the set $\langle A\rangle_{c, i}$ can be explained as follows:

Proposition 2. For any $A \subset U$, one has:

$$
\begin{gathered}
\langle A\rangle_{c, 1}=\left\{u \in U \mid c(u, v) \leq \sup _{a \in A} c(a, v), \forall v \in V\right\}, \\
\langle A\rangle_{c, 2}=\{u \in U|\forall v \in V, \exists a \in A| c(u, v) \leq c(a, v)\}, \\
\langle A\rangle_{c, 3}=\{u \in U|\forall v \in V, \exists a \in A| c(u, v)=c(a, v)\} .
\end{gathered}
$$


Proof By (3.2), one has:

$$
\begin{aligned}
a \notin\langle A\rangle_{c, 1} & \Longleftrightarrow \exists(v, r) \in V \times \mathbb{R}: A \subset[c(., v) \leq r] \text { and } r<c(a, v) \\
& \Longleftrightarrow \exists(v, r) \in V \times \mathbb{R}: \sup _{u \in A} c(u, v) \leq r<c(a, v) \\
& \Longleftrightarrow \exists v \in V: \sup _{u \in A} c(u, v)<c(a, v) .
\end{aligned}
$$

Thus, $a \in\langle A\rangle_{c, 1} \Longleftrightarrow \forall v \in V, c(a, v) \leq \sup _{u \in A} c(u, v)$, and (3.3) holds.

$$
\begin{aligned}
a \notin\langle A\rangle_{c, 2} & \Longleftrightarrow \exists(v, r) \in V \times \mathbb{R}: A \subset[c(., v)<r] \text { and } r \leq c(a, v) \\
& \Longleftrightarrow \exists(v, r) \in V \times \mathbb{R}: c(u, v)<r \leq c(a, v), \forall u \in A \\
& \Longleftrightarrow \exists v \in V: \quad c(u, v)<c(a, v), \forall u \in A .
\end{aligned}
$$

Thus, $a \in\langle A\rangle_{c, 2} \Longleftrightarrow \forall v \in V, \exists u \in A: c(a, v) \leq c(u, v)$, and (3.4) holds.

$$
\begin{aligned}
a \notin\langle A\rangle_{c, 3} & \Longleftrightarrow \exists(v, r) \in V \times \mathbb{R}: A \subset[c(., v) \neq r] \text { and } r=c(a, v) \\
& \Longleftrightarrow \exists v \in V: c(u, v) \neq c(a, v), \forall u \in A .
\end{aligned}
$$

Thus $a \in\langle A\rangle_{c, 3} \Longleftrightarrow \forall v \in V, \exists u \in A: c(a, v)=c(u, v)$, and (3.5) holds.

Remark 3.3. Observe that one cannot remove the real parameter $r$ in the definition of $\langle A\rangle_{c, 4}$.

Example 3.4. We observe the situation in topological vector case. Assume $U$ is a topological vector space with topological dual $V$ and $c$ the standard coupling function. The c-elementary functions are affine continuous functions, and we have:

1. $\left(c, \mathbb{P}_{1}\right)$-elementary sets are $\emptyset, U$ and closed half spaces. Moreover, if $U$ is locally convex, then by Hahn-Banach separation theorem and (3.3), $\langle A\rangle_{c, 1}=\overline{\operatorname{conv}} A$, the closed convex hull of $A$.

2. $\left(c, \mathbb{P}_{2}\right)$-elementary sets are $\emptyset, U$ and half open spaces. The $\left(c, \mathbb{P}_{2}\right)$-hull of a subset of $U$ is its evenly convex hull ([4],[7],...).

3. $\left(c, \mathbb{P}_{3}\right)$-elementary sets are $\emptyset, U$ and complementary set of closed hyperplane. The $\left(c, \mathbb{P}_{3}\right)$-hull of a subset of $U$ is its evenly co-affine hull ([15]). Observe that ([15], corollary 2.2) $A$ is evenly convex if and only if $A$ is evenly co-affine and convex.

4. $\left(c, \mathbb{P}_{4}\right)$-elementary sets are $\emptyset, U$ and closed hyperplane. Moreover, if $U$ is locally convex, then by the Hahn-Banach separation theorem and (3.2), the $\left(c, \mathbb{P}_{4}\right)$-hull of a non empty subset of $U$ is its closed affine hull.

Proposition 3. Let $P$ and $Q$ be two nonvoid subsets of $\mathbb{R}$. Assume that any $(c, P)$-elementary set is $(c, Q)$-regular. Then, $\langle A\rangle_{c, Q} \subset\langle A\rangle_{c, P}, \forall A \subset U$. 
Proof Let $a \notin\langle A\rangle_{c, P}$. By definition, there exists an $(c, P)$-elementary set $E$ such that $A \subset E$ and $a \notin E$. Since $E$ is also $(c, Q)$-regular, it follows from (3.2) that $a \notin\langle A\rangle_{c, Q}$, and we are done.

Corollary 3.5. For any $A \subset U$, one has:

$$
\langle A\rangle_{c, 1} \supset\langle A\rangle_{c, 2} \supset\langle A\rangle_{c, 3} \text { and }\langle A\rangle_{c, 4} \supset\langle A\rangle_{c, 3} .
$$

Proof Let $v \in V$ and $r \in \mathbb{R}$, it is obvious that

$$
\{u \in U \mid c(u, v) \leq r\}=\bigcap_{s>r}\{u \in U \mid c(u, v)<s\} .
$$

Consequently, any $\left(c, \mathbb{P}_{1}\right)$-elementary subset is $\left(c, \mathbb{P}_{2}\right)$-regular, and by Proposition 3 , one has $\langle A\rangle_{c, 1} \supset\langle A\rangle_{c, 2}$ for any $A \subset U$.

It is easy to verify that:

$$
\begin{aligned}
& \{u \in U \mid c(u, v)<r\}=\bigcap_{s \geq r}\{u \in U \mid c(u, v) \neq s\}, \\
& \{u \in U \mid c(u, v)=r\}=\bigcap_{s \neq r}\{u \in U \mid c(u, v) \neq s\},
\end{aligned}
$$

therefore, $\langle A\rangle_{c, 2} \supset\langle A\rangle_{c, 3} \subset\langle A\rangle_{c, 4}$.

We derive from Corollary 3.5, the following comparison between the sets $\mathcal{R}_{c, \mathbb{P}_{i}}(U)$ :

$$
\mathcal{R}_{c, \mathbb{P}_{1}}(U) \subset \mathcal{R}_{c, \mathbb{P}_{2}}(U) \subset \mathcal{R}_{c, \mathbb{P}_{3}}(U) \text { and } \mathcal{R}_{c, P_{4}}(U) \subset \mathcal{R}_{c, \mathbb{P}_{3}}(U) .
$$

Remark 3.6. Observe that

1. $\mathbb{P}_{1} \supset \mathbb{P}_{2}$ and $\mathcal{R}_{c, \mathbb{P}_{1}}(U) \subset \mathcal{R}_{c, \mathbb{P}_{2}}(U)$.

2. $\mathbb{P}_{3} \supset \mathbb{P}_{2}$ and $\mathcal{R}_{c, \mathbb{P}_{3}}(U) \supset \mathcal{R}_{c, \mathbb{P}_{2}}(U)$.

3. $\mathcal{R}_{c, \mathbb{P}_{1}}(U) \subset \mathcal{R}_{c, \mathbb{P}_{3}}(U)$ whereas $\mathbb{P}_{1}$ and $\mathbb{P}_{3}$ are not comparable in the sense of inclusion.

4. In particular, in the case of locally convex vector space, we recover the fact that every closed convex subset is evenly convex.

Proposition 4. Assume that the coupling function $c$ satisfies the property:

$$
\forall v \in V, \exists w \in V \quad \mid \quad-c(., v)=c(., w) .
$$

Then, one has:

$$
\mathcal{R}_{c, \mathbb{P}_{4}}(U) \subset \mathcal{R}_{c, \mathbb{P}_{1}}(U) \subset \mathcal{R}_{c, \mathbb{P}_{2}}(U) \subset \mathcal{R}_{c, \mathbb{P}_{3}}(U) .
$$


Proof By (3.6), we only need to show that $\mathcal{R}_{c, \mathbb{P}_{4}}(U) \subset \mathcal{R}_{c, \mathbb{P}_{1}}(U)$. Let $(v, r) \in V \times \mathbb{R}$. We have

$$
[c(., v)=r]=[c(., v) \leq r] \bigcap[-c(., v) \leq-r] .
$$

By assumption on the coupling function, there exists $w \in V$ such that $-c(., v)=c(., w)$. Consequently,

$$
[c(., v)=r]=[c(., v) \leq r] \bigcap[c(., w) \leq-r] .
$$

We conclude with Proposition 3 ,

Example 3.7. Assume that $U=V=\mathbb{R}^{n}$, and coupling function $c$ is defined by $c(u, v)=\|u-v\|$, where $\|$.$\| is the euclidean norm. The non trivial \left(c, \mathbb{P}_{4}\right)$-elementary sets are spheres (not convex) whereas the non trivial $\left(c, \mathbb{P}_{1}\right)$-elementary sets are closed balls (closed convex). In this case, $\mathcal{R}_{c, \mathbb{P}_{1}}(U)$ and $\mathcal{R}_{c, \mathbb{P}_{4}}(U)$ are not comparable. Observe that in this case assumption (3.7) does not hold.

Proposition 5. For any $A \subset U$, one has $\langle A\rangle_{c, 1}=\left[i_{A}^{\Gamma_{c}} \leq 0\right]$.

Proof By (3.2), one has

$$
\begin{aligned}
a \notin\langle A\rangle_{c, 1} & \Longleftrightarrow \exists v \in V: i_{A}^{c}(v)<c(a, v) \\
& \Longleftrightarrow 0<\sup _{v \in V}\left\{c(a, v)-i_{A}^{c}(v)\right\} \\
& \Longleftrightarrow 0<i_{A}^{\Gamma_{c}}(a) \\
& \Longleftrightarrow 0 \notin\left[i_{A}^{\Gamma_{c}} \leq 0\right] .
\end{aligned}
$$

Thus $\langle A\rangle_{c, 1}=\left[i_{A}^{\Gamma_{c}} \leq 0\right]$.

The following result makes the link between hull of set and $\Gamma$-regularization of function by means of indicator function.

Theorem 3.8. Assume that the coupling function c satisfies the condition:

$$
\forall(v, \beta) \in V \times \mathbb{R}_{+}^{*}, \quad \exists \bar{v} \in V \mid \beta c(., v)=c(., \bar{v}) .
$$

Then for each $A \subset U$ such that $\operatorname{dom} i_{A}^{c} \neq \emptyset$, one has: $i_{A}^{\Gamma_{c}}=i_{\langle A\rangle_{c, 1}}$.

Proof Let $b \in U$.

(1) Assume that $b \notin\langle A\rangle_{c, 1}$. By (3.3), there exists $(v, \epsilon) \in V \times \mathbb{R}_{+}^{*}$ such that $c(b, v)-\sup _{a \in A} c(a, v) \geq$ $\epsilon$. From (3.9), one has:

$$
\forall n \geq 1, \exists v_{n} \in V: n c(., v)=c\left(., v_{n}\right)
$$


Consequently,

$$
n \epsilon \leq c\left(b, v_{n}\right)-\sup _{a \in A} c\left(a, v_{n}\right)=c\left(b, v_{n}\right)-i_{A}^{c}\left(v_{n}\right) \leq i_{A}^{\Gamma_{c}}(b), \quad \forall n \geq 1 .
$$

Therefore $i_{A}^{\Gamma_{c}}(b)=+\infty$.

(2) Assume that $b \in\langle A\rangle_{c, 1}$. By (3.3), one has

$$
c(b, w)-\sup _{a \in A} c(a, w) \leq 0, \quad \forall v \in V .
$$

Thus

$$
i_{A}^{\Gamma_{c}}(b)=\sup _{v \in V}\left\{c(b, v)-\sup _{a \in A} c(a, v)\right\} \leq 0 .
$$

Let $v \in \operatorname{dom} i_{A}^{c}$. By (3.9), one gets

$$
\forall n \geq 1, \exists v_{n} \in V: \frac{1}{n} c(., v)=c\left(., v_{n}\right) .
$$

Consequently,

$$
\frac{1}{n}\left(c(b, v)-\sup _{a \in A} c(a, v)\right)=c\left(b, v_{n}\right)-\sup _{a \in A} c\left(a, v_{n}\right) \leq i_{A}^{\Gamma_{c}}(b), \quad \forall n \geq 1 .
$$

Therefore,

$$
0=\lim _{n \rightarrow+\infty} \frac{1}{n}\left(c(b, v)-\sup _{a \in A} c(a, v)\right) \leq i_{A}^{\Gamma_{c}}(b),
$$

and finally, $i_{A}^{\Gamma_{c}}(b)=0$.

Remark 3.9. Assumption (3.9) is satisfied by coupling functions (1), (3) and (5) of Example 2.2. Coupling functions (2) and (4) of the same example do not satisfy assumption (3.9).

\section{Level set regularization of functions}

In this section, we introduce a notion of $(c, \mathbb{P})$-level set regularization of extended real-valued functions. We show that this level set regularization can be interpreted as bi-conjugacy relative to a couple of dual polarities by decomposition of the closure operator. We then give some other expressions of these regularizations.

\subsection{Definitions and properties}

Definition 4. A function $h: U \rightarrow \overline{\mathbb{R}}$ is said to be $(c, \mathbb{P})$-level regular if all of its sub-level sets are $(c, \mathbb{P})$-regular, i.e $\langle[h \leq r]\rangle_{c, \mathbb{P}}=[h \leq r], \forall r \in \mathbb{R}$. 
We denote $\mathcal{N}_{c, \mathbb{P}}(U)$, the set of $(c, \mathbb{P})$-level regular functions defined on $U$ to $\overline{\mathbb{R}}$. Observe that this set contains the constant function $-\infty$.

Proposition 6. The set $\mathcal{N}_{c, \mathbb{P}}(U)$ is closed under pointwise suprema, i.e given $\left(h_{i}\right)_{i \in I}$ a family of $(c, \mathbb{P})$-level regular functions, then $h:=\sup _{i \in I} h_{i}$ is $(c, \mathbb{P})$-level regular.

Proof Let $r \in \mathbb{R}$. Since $[h \leq r]=\cap_{i \in I}\left[h_{i} \leq r\right]$, the conclusion follows from the fact that any intersection $(c, \mathbb{P})$-regular sets is $(c, \mathbb{P})$-regular.

We define the $(c, \mathbb{P})$-level set regularization of an extended real-valued function as follows.

Definition 5. The $(c, \mathbb{P})$-level set regularization of a function $h: U \rightarrow \overline{\mathbb{R}}$ is the greatest $(c, \mathbb{P})$-level regular minorant of $h$. This function is denoted by $h^{\langle\rangle_{c, \mathbb{P}}}$.

Example 4.1. Assume $U$ is topological vector space with topological dual $V$ and $c$ the standard coupling function. $\left(c, \mathbb{P}_{2}\right)$-level regular functions are evenly quasi-convex functions. Moreover, if $U$ is locally convex then $\left(c, \mathbb{P}_{1}\right)$-level regular functions are lower semi-continuous quasi-convex functions.

Example 4.2. Assume that $U$ is a metric space, $V=\mathscr{C}(U, \mathbb{R})$ a space of continuous functions from $U$ to $\mathbb{R}$, and $c: U \times V \rightarrow \mathbb{R}$ defined by $c(u, v)=v(u)$. A function $h: U \rightarrow \mathbb{R} \cup\{+\infty\}$ is $\left(c, \mathbb{P}_{1}\right)$-level regular if and only if $h$ is lower semi-continuous ([3], corollary 11).

Proposition 7. Any c-elementary function is $\left(c, \mathbb{P}_{i}\right)$-level regular for $i=1,2,3$. More precisely, one has

$$
\Gamma_{c}(U) \subset \mathcal{N}_{c, \mathbb{P}_{1}}(U) \subset \mathcal{N}_{c, \mathbb{P}_{2}}(U) \subset \mathcal{N}_{c, \mathbb{P}_{3}}(U) \text { and } \mathcal{N}_{c, \mathbb{P}_{4}}(U) \subset \mathcal{N}_{c, \mathbb{P}_{3}}(U)
$$

Proof Let $h:=c(., v)-r$ an $c$-elementary function. For any $t \in \mathbb{R}$, we have

$$
[h \leq t]=\{u \in U \mid t+r-c(u, v) \geq 0\},
$$

which is obviously $\left(c, \mathbb{P}_{1}\right)$-elementary set. Therefore $\Gamma_{c}(U) \subset \mathcal{N}_{c, \mathbb{P}}(U)$. The other inclusions follow from (3.6).

Remark 4.3. $c$-elementary functions are not necessary $\left(c, \mathbb{P}_{4}\right)$-level set regular functions. For example, in the topological case, one cannot write a half space as an intersection of affine hyperplanes.

Example 4.4. Let $n \geq 1$, an integer number. Assume that $U=V=\mathbb{R}^{n}$ and $c$ a standard scalar product of $\mathbb{R}^{n}$. Let $h_{1}, h_{2}: \mathbb{R}^{n} \rightarrow \llbracket 0 ; n \rrbracket$ two functions defined by

$$
h_{1}(x)= \begin{cases}0 & \text { if } x=0 \\ \max \left\{i \in \llbracket 1, n \rrbracket \mid x_{i} \neq 0\right\} & \text { if } x \neq 0,\end{cases}
$$




$$
h_{2}(x)= \begin{cases}0 & \text { if } x_{i} \neq 0, \forall i \in \llbracket 1, n \rrbracket \\ \max \left\{i \in \llbracket 1, n \rrbracket \mid x_{i}=0\right\} & \text { else } .\end{cases}
$$

For any $r \in \mathbb{R}$, we have

$$
\begin{aligned}
& {\left[h_{1} \leq r\right]= \begin{cases}\emptyset & \text { if } r<0 \\
\left\{x \in \mathbb{R}^{n} \mid x_{i+1}=\ldots=x_{n}=0\right\} & \text { if } i \leq r<i+1, \quad i=0,1, \ldots, n-1 \\
\mathbb{R}^{n} & \text { if } n \leq r,\end{cases} } \\
& {\left[h_{2} \leq r\right]= \begin{cases}\emptyset & \text { if } r<0 \\
\left\{x \in \mathbb{R}^{n} \mid x_{i+1} \neq 0, \ldots, x_{n} \neq 0\right\} & \text { if } i \leq r<i+1, \quad i=0,1, \ldots, n-1 \\
\mathbb{R}^{n} & \text { if } n \leq r .\end{cases} }
\end{aligned}
$$

It is clear that:

(1) $h_{1}$ is $\left(c, \mathbb{P}_{4}\right)$-level regular. In particular, $h_{1} \in \mathcal{N}_{c, \mathbb{P}_{i}}(U)$, for $i=1,2,3,4$.

(2) $h_{2}$ is $\left(c, \mathbb{P}_{3}\right)$-level regular but not $\left(c, \mathbb{P}_{2}\right)$-level regular since $\left[h_{2} \leq n-1\right]=\left\{x \in \mathbb{R}^{n} \mid x_{n} \neq 0\right\}$ is not convex.

Example 4.5. Let $U=V=\mathbb{R}$, $c$ the standard product of $\mathbb{R}$. The indicator function of $\mathbb{R}^{*}, i_{\mathbb{R}^{*}}$ is $\left(c, \mathbb{P}_{3}\right)$-level regular but not quasi-convex.

\subsection{Decomposition of \langle\rangle$_{c, \mathbb{P}}$}

Let us consider a map $\Delta_{c, \mathbb{P}}: 2^{U} \rightarrow 2^{V \times \mathbb{R}}$ defined by:

$$
\Delta_{c, \mathbb{P}}(A):=\left\{(v, r) \in V \times \mathbb{R} \mid A \subset E_{v, r}^{\mathbb{P}}\right\},
$$

which, we simply denote $\Delta$ in the sequel. Given $\left(A_{i}\right)_{i \in I}$ a family of subsets of $U$, we have

$$
\begin{aligned}
\Delta \bigcup_{i \in I} A_{i} & :=\left\{(v, r) \in V \times \mathbb{R} \mid \bigcup_{i \in I} A_{i} \subset E_{v, r}^{\mathbb{P}}\right\} \\
& =\left\{(v, r) \in V \times \mathbb{R} \mid A_{i} \subset E_{v, r}^{\mathbb{P}}, \quad \forall i \in I\right\} \\
& =\bigcap_{i \in I}\left\{(v, r) \in V \times \mathbb{R} \mid A_{i} \subset E_{v, r}^{\mathbb{P}}\right\} \\
& =\bigcap_{i \in I} \Delta A_{i} .
\end{aligned}
$$

Therefore $\Delta$ is said to be a polarity ([16]). We associate to $\Delta$, its dual polarity $\Delta^{*}: 2^{V \times \mathbb{R}} \rightarrow 2^{U}$ defined by

$$
\Delta^{*}(B)=\bigcup\left\{A \in 2^{U} \mid B \subset \Delta(A)\right\} .
$$

Observe that for each $(v, r) \in V \times \mathbb{R}$ and for each $u \in U$, one has

$$
u \in \Delta^{*}(v, r) \Longleftrightarrow(v, r) \in \Delta(u) \Longleftrightarrow u \in E_{v, r}^{\mathbb{P}},
$$


therefore $\Delta^{*}(v, r)=E_{v, r}^{\mathbb{P}}$. Since $\Delta^{*}$ is a polarity, then we have for each $B \subset V \times \mathbb{R}$,

$$
\Delta^{*}(B)=\Delta^{*}\left(\bigcup_{(v, r) \in B}\{(v, r)\}\right)=\bigcap_{(v, r) \in B} \Delta^{*}(v, r)=\bigcap_{(v, r) \in B} E_{v, r}^{\mathbb{P}}
$$

The operator \langle\rangle$_{c, \mathbb{P}}$ can be decomposed as follows.

Proposition 8. For any $A \subset U$, we have $\left(\Delta^{*} \circ \Delta\right)(A)=\langle A\rangle_{c, \mathbb{P}}$.

Proof Given $A \subset U$, one has

$$
\left(\Delta^{*} \circ \Delta\right)(A)=\Delta^{*}\left(\left\{(v, r) \in V \times \mathbb{R} \mid A \subset E_{v, r}^{\mathbb{P}}\right\}\right)=\bigcap_{A \subset E_{v, r}^{\mathbb{P}}} E_{v, r}^{\mathbb{P}}=\langle A\rangle_{c, \mathbb{P}}
$$

\subsection{Conjugacy associated to polarities $\Delta$ and $\Delta^{*}([16,[17])$}

The conjugate of a function $h: U \rightarrow \overline{\mathbb{R}}$ relative to the polarity $\Delta$ is the function $h^{\Delta}: V \times \mathbb{R} \rightarrow \overline{\mathbb{R}}$ given by

$$
h^{\Delta}(v, r):=\sup _{u \notin \Delta^{*}(v, r)}-h(u)=\sup _{u \notin E_{v, r}^{\mathbb{P}}}-h(u) .
$$

Analogously, the conjugate of a function $k: V \times \mathbb{R} \rightarrow \overline{\mathbb{R}}$ relative to the polarity $\Delta^{*}$ is defined by

$$
k^{\Delta^{*}}(u):=\sup _{(v, r) \notin \Delta(u)}-k(v, r)=\sup _{u \notin E_{v, r}^{\mathbb{P}}}-k(v, r) .
$$

Thus, the bi-conjugacy relative to polarities $\Delta, \Delta^{*}$ of a function $h: U \rightarrow \overline{\mathbb{R}}$ is the function $h^{\Delta \Delta^{*}}: U \rightarrow \overline{\mathbb{R}}$ given by

$$
h^{\Delta \Delta^{*}}(a):=\sup _{(v, r) \notin \Delta(a)} \inf _{u \notin \Delta *(v, r)} h(u)=\sup _{a \notin E_{v, r}^{\mathbb{P}}} \inf _{u \notin E_{v, r}^{\mathbb{P}}} h(u) .
$$

It is well known ([16]) that this conjugacy can be interpreted by means of coupling function $\delta: U \times(V \times \mathbb{R}) \rightarrow \overline{\mathbb{R}}$ defined by

$$
\delta(u,(v, r))=\left\{\begin{array}{lll}
0 & \text { si } & u \notin E_{v, r}^{\mathbb{P}} \\
-\infty & \text { si } & u \in E_{v, r}^{\mathbb{P}} .
\end{array}\right.
$$

More precisely, given a function $h: U \rightarrow \overline{\mathbb{R}}$, we have $h^{\Delta}=h^{\delta}$ and $h^{\Delta \Delta^{*}}=h^{\delta \delta}$.

Theorem $4.6([16])$. The $(c, \mathbb{P})$-level regularization of a function $h: U \rightarrow \overline{\mathbb{R}}$ coincides with bi-conjugacy relative to polarities $\Delta, \Delta^{*}: h^{\langle\rangle_{c, \mathbb{P}}}=h^{\Delta \Delta^{*}}$.

Corollary 4.7. For any subset $A$ of $U$, we have

$$
i_{A}^{\langle\rangle_{c, \mathbb{P}}}=i_{\langle A\rangle_{c, \mathbb{P}}} \text { and } \quad v_{A}^{\langle\rangle_{c, \mathbb{P}}}=v_{\langle A\rangle_{c, \mathbb{P}}}
$$


Proof Let $A \subset U$. Let $a \in A$. It follows from Theorem 4.6 that

$$
i_{A}^{\langle\rangle_{c, \mathbb{P}}}(a)=\sup _{a \notin E_{v, r}^{\mathbb{P}}} \inf _{u \notin E_{v, r}^{\mathbb{P}}} i_{A}(u) .
$$

We distinguish two cases:

- We first assume that $a \notin\langle A\rangle_{c, \mathbb{P}}$. There exists $(v, r) \in V \times \mathbb{R}$ such that $a \notin E_{v, r}^{\mathbb{P}}$ and $A \subset E_{v, r}^{\mathbb{P}}$. Consequently,

$$
\inf _{u \notin E_{v, r}^{\mathbb{P}}} i_{A}(u)=+\infty \text {. }
$$

- Secondly, assume that $a \in\langle A\rangle_{c, \mathbb{P}}$. For all $(v, r) \in V \times \mathbb{R}$ such that $a \notin E_{v, r}^{\mathbb{P}}$, there exists $u \in A$ such that $u \notin E_{v, r}^{\mathbb{P}}$. Consequently,

$$
\begin{aligned}
i_{A}^{\langle\rangle_{c, \mathbb{P}}}(a) & =\sup _{a \notin E_{v, r}^{\mathbb{P}}} \inf _{u \notin E_{v, r}^{\mathbb{P}}} i_{A}(u) \\
& = \begin{cases}0 & \text { if } a \in\langle A\rangle_{c, \mathbb{P}} \\
+\infty & \text { if } a \notin\langle A\rangle_{c, \mathbb{P}}\end{cases} \\
& =i_{\langle A\rangle_{c, \mathbb{P}}}(a) .
\end{aligned}
$$

Analogously, we have

$$
\begin{aligned}
v_{A}^{\langle\rangle_{c, \mathbb{P}}}(a) & =\sup _{a \notin E_{v, r}^{\mathbb{P}}} \inf _{u \notin E_{v, r}^{\mathbb{P}}} v_{A}(u) \\
& = \begin{cases}-\infty & \text { if } a \in\langle A\rangle_{c, \mathbb{P}} \\
+\infty & \text { if } a \notin\langle A\rangle_{c, \mathbb{P}}\end{cases} \\
& =v_{\langle A\rangle_{c, \mathbb{P}}}(a) .
\end{aligned}
$$

Proposition 9. For any function $h: U \rightarrow \overline{\mathbb{R}}$ and for any real number $t$, one has

$$
\left[h^{\langle\rangle_{c, \mathbb{P}}} \leq t\right]=\bigcap_{s>t}\langle[h \leq s]\rangle_{c, \mathbb{P}}
$$

Proof Let $s>t$ and $a \notin\langle[h \leq s]\rangle_{c, \mathbb{P}}$. There exists $(\bar{v}, \bar{r}) \in V \times \mathbb{R}$ such that $a \notin E_{\bar{v}, \bar{r}}^{\mathbb{P}}$ and $[h \leq s] \subset E_{\bar{v}, \bar{r}}^{\mathbb{P}}$. We deduce that

$$
h^{\langle\rangle_{c, \mathbb{P}}}(a)=\sup _{a \notin E_{v, r}^{\mathbb{P}}} \inf _{u \notin E_{v, r}^{\mathbb{P}}} h(u) \geq \inf _{u \notin E_{\bar{v}, \bar{r}}^{\mathbb{P}}} h(u) \geq s>t .
$$

Let $a \notin\left\langle\left[h^{\langle\rangle_{c, \mathbb{P}}} \leq s\right]\right\rangle_{c, \mathbb{P}}$. There exists $s \in \mathbb{R}$ such that $h^{\langle\rangle_{c, \mathbb{P}}}(a)>s>t$. By Theorem 4.6, there exists $(v, r) \in V \times \mathbb{R}$ such that

$$
a \notin E_{v, r}^{\mathbb{P}} \text { and } \inf _{u \notin E_{v, r}^{\mathbb{P}}} h(u)>s,
$$

thus $[h \leq s] \subset E_{v, r}^{\mathbb{P}}$, and finally $a \notin\langle[h \leq s]\rangle_{c, \mathbb{P}}$. 


\subsection{Other expressions of $(c, \mathbb{P})$-level regularizations}

We now give another expression of the $(c, \mathbb{P})$-level regularization of an extended real-valued function $h$. These expressions give the value of the $(c, \mathbb{P})$-level regularization of $h$ at a given point. Given $h: U \rightarrow \overline{\mathbb{R}}$ and $a \in U$, we define sets $\mathcal{I}_{h}(a)$ and $\mathcal{J}_{h}(a)$ by:

$$
\mathcal{I}_{h}(a):=\left\{t \in \mathbb{R} \mid a \notin\langle[h \leq t]\rangle_{c, \mathbb{P}}\right\} \text { and } \mathcal{J}_{h}(a)=\left\{t \in \mathbb{R} \mid a \in\langle[h \leq t]\rangle_{c, \mathbb{P}}\right\}
$$

Sets $\mathcal{I}_{h}(a)$ and $\mathcal{J}_{h}(a)$ are two intervals of $\mathbb{R}$ such that $\mathcal{I}_{h}(a) \cap \mathcal{J}_{h}(a)=\emptyset$ and $\mathcal{I}_{h}(a) \cup \mathcal{J}_{h}(a)=\mathbb{R}$. Moreover, for any $(r, s) \in \mathcal{I}_{h}(a) \times \mathcal{J}_{h}(a)$, we have $r<s$. We deduce that $\sup \mathcal{I}_{h}(a)=\inf \mathcal{J}_{h}(a)$.

\section{Proposition 10.}

$$
h^{\langle\rangle_{c, \mathbb{P}}}(a)=\sup \left\{t \in \mathbb{R}: a \notin\langle[h \leq t]\rangle_{c, \mathbb{P}}\right\}=\inf \left\{t \in \mathbb{R}: a \in\langle[h \leq t]\rangle_{c, \mathbb{P}}\right\} .
$$

Proof Let $t \in \mathcal{I}_{h}(a)$. There exists $(v, r) \in V \times \mathbb{R}$ such that $a \notin E_{v, r}^{\mathbb{P}}$ and $[h \leq t] \subset E_{v, r}^{\mathbb{P}}$. Therefore

$$
\inf _{u \notin E_{v, r}^{\mathbb{P}}} h(u) \geq t \text { and so } \sup _{a \notin E_{v, r}^{\mathbb{P}}} \inf _{u \notin E_{v, r}^{\mathbb{P}}} h(u) \geq t .
$$

By Theorem 4.6, one gets $h^{\langle\rangle_{c, \mathbb{P}}}(a) \geq t$. Hence $h^{\langle\rangle_{c, \mathbb{P}}}(a) \geq \sup \mathcal{I}_{h}(a)$. Conversely, let $t<h^{\langle\rangle_{c, \mathbb{P}}}(a)$, then $a \notin\left[h^{\langle\rangle_{c, \mathbb{P}}} \leq t\right]$ and by Proposition 9 there exists $s>t$ such that $a \notin\langle[h \leq s]\rangle_{c, \mathbb{P}}$. Consequently, $\sup \mathcal{I}_{h}(a) \geq s>t$. Hence $\sup \mathcal{I}_{h}(a) \geq h^{\langle\rangle_{c, \mathbb{P}}}(a)$.

\section{Applications to an optimization problem: sub-level set duality}

Let us consider the following minimization problem:

$$
\min _{x} f(x), \quad \text { s.t } \quad x \in X,
$$

where $X$ is a nonempty set and $f: X \rightarrow \mathbb{R} \cup\{+\infty\}$ is an extended real-valued function.

\subsection{Level set perturbational duality}

We consider a perturbation function $F: X \times U \rightarrow \overline{\mathbb{R}}$ satisfying

$$
\exists a \in U: F(., a)=f(.) \text {. }
$$

We associate to $F$ a valued function $h: U \rightarrow \overline{\mathbb{R}}$ defined by

$$
h(u):=\inf _{x \in X} F(x, u)
$$


We denote by $\alpha$ the optimal value of $(\mathscr{P})$. It is obvious that $\alpha=h(a)$.

The perturbational dual of $\mathscr{P}([16)$ is given by

$$
\max _{(v, r)}-h^{\Delta}(v, r) \quad \text { s.t } \quad a \notin E_{v, r}^{\mathbb{P}} .
$$

We denote by $\beta$ the optimal value of (D). By definition, one has

$$
-\infty \leq \beta:=\sup (\mathscr{D})=h^{\Delta \Delta^{*}}(a) \leq h(a)=: \alpha=\inf (\mathscr{P}) \leq+\infty .
$$

Thus, the weak duality holds. The following theorem gives a necessary and sufficient condition to assure the strong duality.

Theorem 5.1. The following statements are equivalent:

(1) The strong duality holds for $(\mathscr{P})$ i.e $\inf (\mathscr{P})=\max (\mathscr{D})$,

(2) $a \notin\langle[h<\alpha]\rangle_{c, \mathbb{P}}$.

Proof. Assume that (1) holds. There exists $(\bar{v}, \bar{r}) \in V \times \mathbb{R}$ such that

$$
a \notin E_{\bar{v}, \bar{r}}^{\mathbb{P}} \text { and } \alpha=h(a)=-h^{\Delta}(\bar{v}, \bar{r}):=\inf _{u \notin E_{\bar{v}, \bar{r}}^{\mathbb{r}}} h(u) .
$$

We deduce that $[h<\alpha] \subset E_{\bar{v}, \bar{r}}^{\mathbb{P}}$. Thus $a \notin\langle[h<\alpha]\rangle_{c, \mathbb{P}}$. Conversely, assume that (2) holds. There exists $(\bar{v}, \bar{r}) \in V \times \mathbb{R}$ such that $a \notin E_{\bar{v}, \bar{r}}^{\mathbb{P}}$ and $[h<\alpha] \subset E_{\bar{v}, \bar{r}}^{\mathbb{P}}$. Therefore

$$
\inf _{u \notin E \mathbb{\overline { v }}, \bar{r}} h(u) \geq \alpha \geq \beta .
$$

Remember that

$$
\beta:=\sup _{a \notin E_{v, r}^{\mathbb{P}}}-h^{\Delta}(v, r)=\sup _{a \notin E_{v, r}^{\mathbb{P}}} \inf _{u \notin E_{v, r}^{\mathbb{P}}} h(u) .
$$

Thus

$$
\beta \geq \inf _{u \notin E_{\bar{v}, \bar{r}}^{\mathbb{P}}} h(u)=-h^{\Delta}(\bar{v}, \bar{r}) \geq \alpha \geq \beta .
$$

Hence $\beta=-h^{\Delta}(\bar{v}, \bar{r})=\alpha$.

Theorem 5.1 is interesting in evenly convex case which is used in economic theory.

\subsection{Evenly quasi-convex duality $([1],[7],[11,[12],[17])$}

We assume $X$ and $U$ are topological vector spaces, $V=U^{*}$ the topological dual of $U, c=\langle$,$\rangle the$ standard coupling function between $U$ and $U^{*}$. 
Corollary 5.2. Assume that function $F: X \times U \rightarrow \overline{\mathbb{R}}$ is quasi-convex and for each $x \in X$, $F(x,):. U \rightarrow \overline{\mathbb{R}}$ is upper semi-continuous. One has:

$$
\inf (\mathscr{P})=\max _{u^{*} \in U^{*}} \inf _{\substack{(x, u) \in X \times U \\\left\langle u-a, u^{*}\right\rangle \geq 0}} F(x, u) .
$$

Proof Since $F$ is quasi-convex and for each $x \in X, F(x,$.$) is upper semi-continuous then h$ is quasi-convex and upper semi-continuous. Consequently, $[h<\alpha]$ is open convex set and so it is evenly convex. As $a \notin[h<\alpha]$, it results from Theorem 5.1 that

$$
\inf (\mathscr{P})=\max (\mathscr{D})=\max _{r-\left\langle a, u^{*}\right\rangle \leq 0} \inf _{r-\left\langle u, u^{*}\right\rangle \leq 0} h(u)=\max _{u^{*} \in U^{*}} \inf _{\left\langle u, u^{*}\right\rangle \geq\left\langle a, u^{*}\right\rangle} h(u),
$$

where the last equality follows from the fact that for each $u^{*} \in U^{*}$, function $k_{u^{*}}: \mathbb{R} \rightarrow \overline{\mathbb{R}}$ defined by $k_{u^{*}}(r)=\inf _{r-\left\langle u, u^{*}\right\rangle \leq 0} h(u)$ is not decreasing.

Corollary 5.3. Assume that function $F: X \times U \rightarrow \overline{\mathbb{R}}$ is quasi-convex and for each $x \in X$, $F(x,):. U \rightarrow \overline{\mathbb{R}}$ is upper semi-continuous. One has:

$$
\inf (\mathscr{P})=\max _{u^{*} \in U^{*}} \inf _{\substack{(x, u) \in X \times U \\\left\langle u-a, u^{*}\right\rangle=0}} F(x, u)
$$

Proof We know that under these assumptions on $F,[h<\alpha]$ is convex open set, therefore it is $\left(\langle\rangle,, \mathbb{R}^{*}\right)$-regular. Since $a \notin[h<\alpha]$, it results from Theorem 5.1 that

$$
\begin{aligned}
\inf (\mathscr{P}) & =\max (\mathscr{D}) \\
& =\max _{\substack{\left(u^{*}, r\right) \in U^{*} \times \mathbb{R} \\
\left\langle a, u^{*}\right\rangle=r}} \inf _{u \in U, U} h(u) \\
& =\max _{\left.u^{*} \in U^{*}\right\rangle=r} \max _{\substack{r \in \mathbb{R} \\
\left\langle a, u^{*}\right\rangle=r}} \inf _{u \in U} h\left(u, u^{*}\right\rangle=r \\
& =\max _{u^{*} \in U^{*}} \inf _{\substack{u \in U \\
\left\langle u-a, u^{*}\right\rangle=0}} h(u) \\
& =\max _{u^{*} \in U^{*}} \inf _{\substack{(x, u) \in X \times U \\
\left\langle u-a, u^{*}\right\rangle=0}} F(x, u) \quad \text { by definition of } h .
\end{aligned}
$$

\section{Conclusion}

In this work, we introduced a closure operator by means of coupling function and a subset of $\mathbb{R}$. This operator allowed us to define a hull of sets and level set regularization of extended real-valued functions. By decomposition of closure operator, we showed that a level set regularization of any 
extended real-valued function coincides with its bi-conjugacy relative to a couple of dual polarities. We derive an analytic expression of a level set regularization of extended real-valued function. Our results are applied to derive a strong duality for a minimization problem.

Acknowledgements The authors are grateful to the anonymous referees and the editor for their constructive comments which have contributed to the final presentation of the paper. 


\section{References}

[1] Crouzeix,J-P.: Contributions l'étude des fonctions quasiconvexes. Thesis. University of Clermont-Ferrand, France (1977)

[2] Dolecki, S. and Kurcyusz, S.: On $\phi$-convexity in extremal problems. SIAM J. Control Optim. 16, 277-300 (1978)

[3] Elias, L.M. and Martínez-Legaz, J.E.: A simplified conjugation scheme for lower semicontinuous functions. Optimization, 65(4):751-763 (2016)

[4] Fenchel,W.: A remark on convex sets and polarity. Comm. Sém. Math. Univ. Lund[Medd. Lunds Univ. Mat. Sem.], 82-89 (1952)

[5] Flores-Bazán, F.: On a notion of subdifferentiability for non-convex functions. Optimization, $33(1): 1-8(1995)$

[6] Guillaume,S. and Volle,M.: Level set relaxation, epigraphical relaxation and conditioning in optimization. Positivity, 19:769-795 (2015)

[7] Martínez-Legaz, J.: Generalized Convex Duality and its Economic Applicatons. Nonconvex Optimization and Its Application, Handbook of generalized convexity and generalized monotonicity. Springer, New York, (2005)

[8] Moreau, J.J.: Inf-convolution, sous-additivité, convexité des fonctions numériques. J. Math. Pures Appl., 49:109-154 (1970)

[9] Penot,J.P.: What is quasiconvex analysis? Optimization, 47:35-110, (2000)

[10] Penot,J.P.: Conjugacies adapted to lower semicontinuous functions. Optimization, 64(3):473$494(2015)$

[11] Penot, J.P. and Volle,M.: On quasi-convex duality. Math. Oper. Res, 15:4597-625, (1990)

[12] Penot, J.P. and Volle,M.: Surrogate programming and multipliers in quasi-convex programming. SIAM J. Control Optim, 42(6):1994-2003, (2004)

[13] Rockafellar,R.T.: Conjugate Duality and optimization, SIAM (1974)

[14] Rubinov,A.: Abstract Convexity and Global Optimization. Nonconvex Optimization and Its Application. Springer US, (2000)

[15] Singer, I.: Abstract convex analysis. Canadian Mathematics Series of Monographs and Texts. A wiley Interscience,(1997) 
[16] Volle,M.: Conjugaison par tranches. Annali di Matematica pura ed applicata, CXXXIX(IV):279-312, (1985).

[17] Volle,M.: Conjugaison par tranche et dualité de toland. Optimization, 18(5):633-642 (1987) 